Check for updates

Cite this: Soft Matter, 2018, 14,255

Received 26th October 2017, Accepted 7th December 2017

DOI: $10.1039 / c 7 s m 02113 e$

rsc.li/soft-matter-journal

\section{Unravelling cationic cellulose nanofibril hydrogel structure: NMR spectroscopy and small angle neutron scattering analyses $\uparrow+$}

\author{
James C. Courtenay, (D) ab Susana M. Ramalhete, ${ }^{c}$ William J. Skuze, ${ }^{b}$ Rhea Soni, ${ }^{c}$ \\ Yaroslav Z. Khimyak, (D) ${ }^{c}$ Karen J. Edler (iD ${ }^{a b}$ and Janet L. Scott (D) ${ }^{a b}$
}

\begin{abstract}
Stiff, elastic, viscous shear thinning aqueous gels are formed upon dispersion of low weight percent concentrations of cationically modified cellulose nanofibrils (CCNF) in water. CCNF hydrogels produced from cellulose modified with glycidyltrimethylammonium chloride, with degree of substitution (DS) in the range 10.6(3)-23.0(9)\%, were characterised using NMR spectroscopy, rheology and small angle neutron scattering (SANS) to probe the fundamental form and dimensions of the CCNF and to reveal interfibrillar interactions leading to gelation. As DS increased CCNF became more rigid as evidenced by longer Kuhn lengths, 18-30 nm, derived from fitting of SANS data to an elliptical cross-section, cylinder model. Furthermore, apparent changes in CCNF cross-section dimensions suggested an "unravelling" of initially twisted fibrils into more flattened ribbon-like forms. Increases in elastic modulus (7.9-62.5 Pa) were detected with increased DS and ${ }^{1} \mathrm{H}$ solution-state NMR $T_{1}$ relaxation times of the introduced surface $-\mathrm{N}^{+}\left(\mathrm{CH}_{3}\right)_{3}$ groups were found to be longer in hydrogels with lower DS, reflecting the greater flexibility of the low DS CCNF. This is the first time that such correlation between DS and fibrillar form and stiffness has been reported for these potentially useful rheology modifiers derived from renewable cellulose.
\end{abstract}

\section{Introduction}

Cellulose fibres can be processed into an array of nanoparticles including nanocrystals, nanowhiskers, and micro- and nanofibrils. ${ }^{1}$ An array of mechanical nanofibrillation methods, enzymatic pretreatments and chemical modifications have been applied to yield so-called 'nanofibrillated' cellulose, as summarised in the comprehensive review by Kalia $e t$ al. ${ }^{2}$ First described by Rånby in $1951,{ }^{3}$ cellulose nanocrystals and nanowhiskers are usually prepared by strong acid hydrolysis of cellulose fibres to yield stiff rod-like, crystalline cellulose nanoparticles with diameters ranging from 2 to $20 \mathrm{~nm}^{4-6}$ These nanoparticles have an axial elastic modulus greater than that of Kevlar, and thus are of interest in the composite materials industry, ${ }^{7}$ however, up to $80 \%$ of the cellulose mass is lost during the hydrolysis process.

\footnotetext{
${ }^{a}$ Centre for Sustainable Chemical Technologies, University of Bath, Bath BA2 7AY, UK. E-mail: j.l.scott@bath.ac.uk

${ }^{b}$ Department of Chemistry, University of Bath, Bath BA2 7AY, UK

${ }^{c}$ School of Pharmacy, University of East Anglia, Norwich Research Park, Norwich, NR4 $7 T G, U K$

$\dagger$ Experimental work was conducted by JCC (CCNF synthesis and characterisation; conductometry, NMR, SANS and TEM) with the support of WJS (conductometry and rheology). SMR lead the NMR studies with the support of RS and JCC.

\# Electronic supplementary information (ESI) available: Material characterisation, SANS fitting parameter information and NMR analyses. See DOI: 10.1039/c7sm02113e
}

As an alternative method to produce nanoparticulate cellulose Saito et al. demonstrated that oxidation, followed by relatively gentle shear, led to aqueous dispersions of oxidised cellulose nanofibrils with a cross-section of $3-5 \mathrm{~nm}^{8}{ }^{8,9}$ This method results in conversion of between 80 and $90 \%$ of the input cellulose into the product nanofibrils.

Well dispersed cellulose nanocrystals and nanofibrils have been widely used to form hydrogels, ${ }^{10,11}$ which are cross-linked by physical interactions (van der Waals forces), chain entanglement, hydrogen bonds, and hydrophobic and electrostatic interactions. In addition to the significantly enhanced yields of cellulose nanofibrils (CNF) achieved by dispersion following chemical modification, such as oxidation, hydrogels can be formed by dispersion of small quantities of CNF in water. As the nanofibrils have a very large aspect ratio and retain a degree of fibril flexibility, these can be induced to form gels at low weight percent inclusion. ${ }^{12}$ Many reports have appeared of chemical modification of hydroxyl groups exposed on the surface of cellulose materials, leading to new applications for cellulose as a biomaterial, ${ }^{13}$ drug delivery vehicle ${ }^{14}$ or water purifying agent ${ }^{15}$ and there is a wide array of chemistry that can be applied to derivatisation of exposed hydroxyl groups including: oxidation, ${ }^{16}$ grafting of cationic tetralkylammonium groups, ${ }^{17}$ sulfonation, ${ }^{18}$ silylation, ${ }^{19}$ acylation, ${ }^{20}$ grafting of a range of substituents through ester linkages ${ }^{21}$ (generated by reaction 


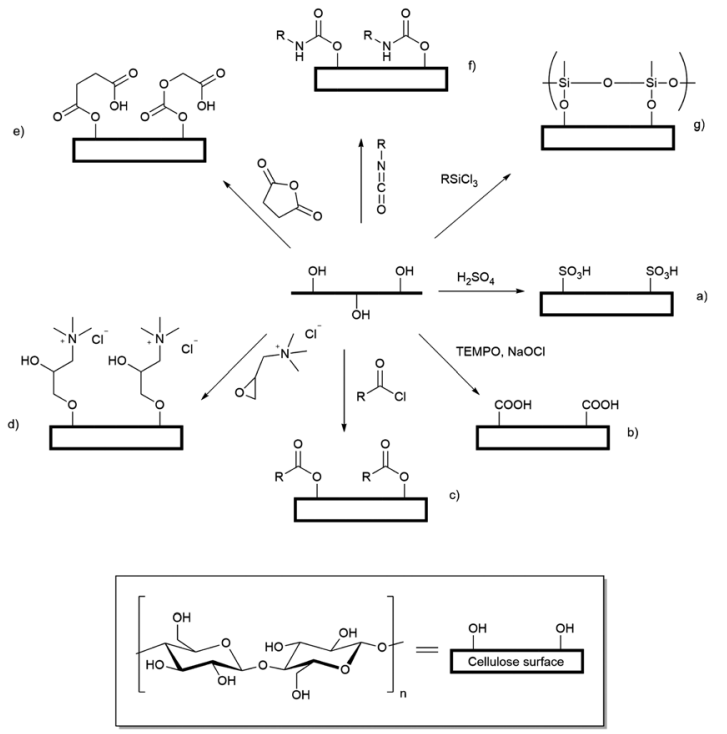

Scheme 1 Examples of some chemical modifications possible by reaction of the hydroxyl groups exposed on the surface of cellulose nanofibrils: (a) sulfonation; (b) (2,2,6,6-tetramethylpiperidin-1-yl)oxyl (TEMPO) mediated oxidation; (c) ester formation by reaction with acid chlorides; (d) grafting of tetra-alkylammonium groups by reaction with glycidyltrimethylammonium chloride (GTMAC); (e) grafting via ester linkages generated by reaction with acid anhydrides; ( $f$ ) formation of urethanes by reaction with isocyanates; and (g) silylation.

with acid anhydrides, ${ }^{22}$ or chlorides $^{23}$ ), and formation of polyurethane linkages by reaction with isocyanates ${ }^{24}$ (Scheme 1). Modification of the surface exposed hydroxyl groups leads to disruption of the non-covalent interactions between bundled cellulose fibrils, allowing dispersion of the individualised CNF in water leading to formation of hydrogels. ${ }^{25}$ The driving force for the formation of modified cellulose hydrogels is the molecular self-assembly of nanofibrils through electrostatic repulsion of charged functional groups, hydrophobic interactions and hydrogen bonds. $^{26}$

Two simple to execute and easily scaled up reactions allow production of charged cellulose fibrils: derivatisation with GTMAC $^{17,27}$ or TEMPO-mediated oxidation ${ }^{8,9,28}$ provide positively charged tetra-alkylammonium groups and potentially negatively charged carboxylate groups, respectively. Both modifications enhance the dispersibility of the functionalised CNF in water. For brevity these modified $\mathrm{CNF}$ are henceforth referred to as cationic cellulose nanofibrils (CCNF) and oxidised cellulose nanofibrils (OCNF).

A wide range of techniques has been used to characterise the surface modification of these nanofibrils: infrared (IR) spectroscopy and zeta-potential measurements are commonly used to identify the new functional groups present and determine whether the surface charge has changed..$^{17,29-31}$ The degree of derivatisation (oxidation or substitution) is determined through an acid-base titration for oxidised cellulose and conductometric titration with $\mathrm{AgNO}_{3}$ for cationic cellulose. ${ }^{16,27,32}$ Recently, Kono \& Kusumoto demonstrated that the degree of substitution (DS) on CCNFs could be calculated through quantitative ${ }^{13} \mathrm{C}$ solid-state
NMR studies, thus supporting conductometric measurements. ${ }^{33}$ Furthermore, small angle neutron scattering can be used to determine the cross-section shape and dimensions of the nanofibrils as well the structure of hydrogels. ${ }^{34,35}$

While the structure of OCNF and hydrogels formed from OCNF dispersions have been extensively probed, ${ }^{8,9,20,28,36,37}$ CCNF based hydrogels are less well described. These would not be predicted to be interchangeable; CCNF bear fixed positive charges in the form of tetra-alkylammonium groups, while the carboxylate moieties on OCNF may be protonated, or coordinated with a range of metal ions. In spite of the body of research into the characterisation of cationic cellulose nanofibrils, e.g. quantifying DS and effect of DS on gelation and CCNF dimensions, ${ }^{17,27,38}$ there is little understanding, at the molecular level, of CCNF surfaces and inter-nanofibril interactions. Herein we describe the influence of DS on physical interactions of CCNF probed using a combination of NMR spectroscopy, rheology and small angle neutron scattering to provide greater understanding of these nanoparticle surfaces and interactions.

\section{Materials and methods}

$\alpha$-Cellulose powder (C8002), sodium hydroxide pellets $(\geq 98 \%)$, glycidyltrimethylammonium chloride ( $\geq 90 \%$ ), $0.1 \mathrm{M} \mathrm{AgNO}$ aqueous solution $(\geq 95 \%)$, and $\mathrm{HCl}$ (reagent grade) were purchased from Sigma-Aldrich UK and used as received. Aqueous solutions of $\mathrm{AgNO}_{3}, \mathrm{NaOH}$ and $\mathrm{HCl}$ were made up to the required concentrations with deionised (DI) water. Sodium polyethylene sulfonate ( $1 \mathrm{mM}$, average $M_{\mathrm{w}} 9000-11000 \mathrm{~g} \mathrm{~mol}^{-1}$ ) solution was supplied by Carisbrooke. Deuterium oxide $\left(\mathrm{D}_{2} \mathrm{O}\right)$ was purchased from Goss Scientific.

\section{Surface modification by derivatisation}

Cationic cellulose. Following the semi-dry procedure described by Zaman, ${ }^{17} \alpha$-cellulose was activated by mixing with 5 wt $\%$ (relative to $\alpha$-cellulose weight, corrected for residual moisture content, determined by thermogravimetric analysis (TGA) to be ca. $5 \%$ ) of powdered $\mathrm{NaOH}$ using a mortar and pestle for $5 \mathrm{~min}$. The mixture was transferred to a polyethylene bag followed by addition of $36 \mathrm{wt} \%$ DI water (relative to $\alpha$-cellulose dry weight) and an appropriate measured quantity of GTMAC ( $\alpha$-cellulose : GTMAC molar ratios of $3: 1,2: 1,1: 1,1: 2$ and $1: 3$ ) was added dropwise to the mixture, the paste was kneaded to homogenise the reactants and reacted at $65{ }^{\circ} \mathrm{C}$ for $75 \mathrm{~min}$ followed by purification by dialysis $\left(M_{\mathrm{W}}\right.$ cut-off $12400 \mathrm{~g} \mathrm{~mol}{ }^{-1}$ ) against copious quantities of DI water (refreshed regularly) for $72 \mathrm{~h}$. The product was concentrated by centrifugation, the supernatant discarded and a sample freeze dried and accurately weighed to determine solids content.

Conductometric titration. Two conductometric titration methods were used to determine DS, the molar percentage of tetra-alkylammonium chloride groups per anhydroglucose unit (AGU) in the bulk product: (1) titration of chloride ions with $\mathrm{AgNO}_{3(\mathrm{aq})}$, as described previously ${ }^{13}$ and (2) using a Mütek Particle Charge Detector (BTG) measuring the streaming potential of $0.25 \mathrm{wt} \%$ cationic cellulose in DI water titrated 
against a $1 \mathrm{mM}$ sodium polyethylene sulfonate solution, following a method previously described for poly(diallyldimethylammonium chloride), ${ }^{39}$ repeated for volumes of $0.25,0.50$ and $1.0 \mathrm{~cm}^{3}$ of the CCNF suspensions.

Titre volume $\left(V_{\mathrm{t}}\right)$, titrant concentration $\left(C_{\mathrm{t}}\right)$, molar mass of AGU $\left(M_{\mathrm{c}}=162.15 \mathrm{~g} \mathrm{~mol}^{-1}\right)$, molar mass of substituent group $\left(M_{\mathrm{s}}=151.63 \mathrm{~g} \mathrm{~mol}^{-1}\right)$, cationic cellulose suspension volume $\left(V_{\mathrm{c}}\right)$, suspension concentration $\left(C_{\mathrm{c}}\right)$ from the Mütek titration and TGA water content values $\left(T_{\mathrm{w}}\right)$ were used to determine the DS of the sample. Substituent mole equivalent per $\mathrm{g}\left(S_{\mathrm{eq}} \mathrm{g}^{-1}\right)$ was determined from plotting molar charge equivalent (eq.) against weight of cellulose $(\mathrm{g})$ with the gradient as eq. $\mathrm{g}^{-1}$. The degree of substitution can be calculated using eqn (1):

$\mathrm{DS} \%=\left[\frac{\left(S_{\mathrm{eq}} \mathrm{g}^{-1} \times M_{\mathrm{c}}\right)}{\left(1-S_{\mathrm{eq}} \mathrm{g}^{-1} \times M_{\mathrm{s}}\right)}\right] \times 100=\left[\frac{(\mathrm{mol} \text { substituents })}{(\mathrm{mol} \mathrm{AGU})}\right] \times 100$

Characterisation. FTIR spectra for $\alpha$-cellulose and CCNF (DS $=23.0(9) \%$ ) powders were obtained on a Perkin Elmer Spectrum 100 spectrometer with a universal ATR sampling accessory; ten scans were acquired in the range $4000-600 \mathrm{~cm}^{-1}$ (Fig. S1, ESI $\ddagger$ ). Prominent bands at $1440 \mathrm{~cm}^{-1}$ and $1483 \mathrm{~cm}^{-1}$ were attributed to the $\mathrm{CH}_{2}$ bending mode and methyl groups of the moieties arising from GTMAC reaction in accordance with data previously published. ${ }^{17}$

Transmission electron microscope (TEM) images of CCNF were obtained on a JEOL JEM-2100Plus instrument with an operating voltage of $200 \mathrm{kV}$. Before image acquisition, samples were deposited on copper grid and negatively stained with uranyl acetate.

\section{CCNF stabilised hydrogels}

Hydrogel formation. Bulk, never-dried CCNF dispersion (6.40 wt\%, concentration accurately measured) was diluted with DI water to yield $4 \mathrm{wt} \%, 2 \mathrm{wt} \%$ and $1 \mathrm{wt} \%$ dispersions which were homogenised using an IKAT18 basic Ultra-Turrax high speed homogeniser at $13500 \mathrm{rpm}$ for $15 \mathrm{~min}$. Dispersion to form hydrogels was completed by sonication using a VibraCell ultrasonicator (Sonics and Materials) at a power output of $45 \mathrm{~W} \mathrm{~cm}^{-2}$ using $1 \mathrm{~s}$ on/off pulses for $2 \mathrm{~min}$. Dispersions were stored at $4{ }^{\circ} \mathrm{C}$ until required.

Hydrogel rheology. Dynamic rheology of the CCNF hydrogels was studied using a Gemini Advanced Cone and Plate Rheometer (Bohlin Instruments); distance between cone and plate $150 \mu \mathrm{m}$ and cone angle $4^{\circ}$. CCNF 2 wt $\%$ suspensions with DS ranging between $10.6-23.0 \%$ were analysed with gel samples placed on the $40 \mathrm{~mm}$ diameter plate and allowed to relax for $5 \mathrm{~min}$ prior to measurement. An oscillatory shear sweep (0.01-10 Hz) was used to determine the dynamic viscosity $\left(\eta^{\prime}\right)$, elastic storage modulus $\left(G^{\prime}\right)$ and loss modulus $\left(G^{\prime \prime}\right)$. A thixotropic loop was performed by measuring the shear viscosity over a ramp of $0.01-150 \mathrm{~s}^{-1}$ followed by return to $0.01 \mathrm{~s}^{-1}$ with zero strain applied.

\section{Cationic cellulose nanofibril characterisation}

Nuclear magnetic resonance (NMR) spectroscopy. Solid-state NMR spectroscopy was used to detect the presence of functional groups on the CCNF. These experiments were conducted using a Bruker Avance III spectrometer equipped with a $4 \mathrm{~mm}$ wide bore probe and operating at ${ }^{1} \mathrm{H}$ and ${ }^{13} \mathrm{C}$ frequencies of 400.23 and 100.65 MHz, respectively. Hydrogels were transferred into Kel-F inserts using a needle and syringe. Lyophilised cationic and $\alpha$-cellulose powders were packed directly into zirconia rotors. ${ }^{1} \mathrm{H}-{ }^{13} \mathrm{C}$ cross-polarisation magic angle spinning (CP/MAS) NMR experiments of hydrogels were performed using recycle delays of $20 \mathrm{~s}, 1094$ scans and MAS rates of $5 \mathrm{kHz} .{ }^{1} \mathrm{H}-{ }^{13} \mathrm{C}$ CP/MAS NMR experiments of reference powders were performed using recycle delays of $20 \mathrm{~s}, 256$ scans and MAS rates of $5 \mathrm{kHz}$ (Fig. S5, ESI $\$$ ). CP kinetics curves were measured using contact times from 0.005 to $10 \mathrm{~ms}$ and were fitted to eqn (2): ${ }^{40}$

$$
I(t)=I_{0}\left(1-T_{I S} / T_{1 \rho}^{I}\right)^{-1}\left[\exp \left(-\frac{t}{T_{1 \rho}^{I}}\right)-\exp \left(-\frac{t}{T_{I S}}\right)\right]
$$

where $I(t)$ is the peak intensity, $I_{0}$ is the absolute intensity, $T_{I S}$ is the cross-polarisation time constant and $T_{1 \rho}^{I}$ is the longitudinal relaxation time in the rotating frame. The degree of substitution was calculated using eqn (3), in accordance with the methodology published by Kono et al. ${ }^{33}$

$$
\text { Degree of substitution }=\frac{\frac{I_{\mathrm{C} 10}}{3}}{I_{\mathrm{C} 1}} \times 100
$$

where $I_{\mathrm{C} 10}$ and $I_{\mathrm{C} 1}$ are the ${ }^{13} \mathrm{C}$ peak intensities of signals due to the three methyl carbon atoms (C10) and AGU anomeric carbon atom (C1), respectively, from the fitted curves. All experiments were conducted at $25{ }^{\circ} \mathrm{C}$ except where otherwise stated. HartmannHahn conditions were optimised using hexamethylbenzene and all spectra were referenced to external tetramethylsilane (TMS).

High-resolution magic angle spinning (HR-MAS) NMR experiments were conducted using a Bruker Avance III spectrometer operating at a ${ }^{1} \mathrm{H}$ frequency of $800.23 \mathrm{MHz}$ equipped with a $4 \mathrm{~mm}$ probe, using Kel-F inserts as above. ${ }^{1} \mathrm{H}$ HR-MAS NMR spectra were acquired using a recycle delay of $5 \mathrm{~s}$ and MAS rates ranging from 2 to $10 \mathrm{kHz} .{ }^{1} \mathrm{H}$ longitudinal relaxation times $\left(T_{1}\right)$ experiments were performed using an inversion recovery pulse sequence with a recycle delay of $10 \mathrm{~s}$. Sixteen points were recorded at variable time delays ranging from 0.1 to $20 \mathrm{~s} .{ }^{1} \mathrm{H} T_{1}$ times were calculated from fitting the evolution of intensities to the mono-exponential function, eqn (4):

$$
M_{z}(\tau)=M_{0} \times\left[1-2 \mathrm{e}^{\left(\frac{-\tau}{T_{1}}\right)}\right]
$$

where $M_{z}$ is the $z$-component of magnetisation, $M_{0}$ is the equilibrium magnetisation and $\tau$ is the time delay.

Solution-state NMR experiments were carried out using a Bruker Avance I spectrometer operating at a ${ }^{1} \mathrm{H}$ frequency of 499.69 $\mathrm{MHz}$ equipped with a $5 \mathrm{~mm}$ triple resonance probe. Samples of hydrogels $(500 \mu \mathrm{L})$ were transferred into NMR tubes using a needle and syringe. Variable temperature experiments were carried out in the range between 5 and $45{ }^{\circ} \mathrm{C} .{ }^{1} \mathrm{H} T_{1}$ relaxation times were measured using an inversion recovery 
pulse sequence with a recycle delay of $10 \mathrm{~s}$. Sixteen points were recorded at variable time delays ranging from 0.1 to $20 \mathrm{~s}$.

Zeta-potential characterisation. A DTS1070 Zetasizer cell was filled with $0.5 \mathrm{~mL}$ of dilute CCNF dispersions, $\sim 0.1 \mathrm{wt} \%$ and placed in a Zetasizer Nanoseries. Electrophoresis was induced in the sample by applying an electric field across the suspension of CCNF. The electrophoretic mobility of the CCNF in the electric field was used to calculate the zeta-potential by applying the Henry eqn (5):

$$
U_{\mathrm{E}}=\frac{2 \varepsilon z f(K \alpha)}{3 \eta}
$$

where $z$ is zeta-potential, $U_{\mathrm{E}}$ is electrophoretic mobility, $\varepsilon$ is the dielectric constant, $\eta$ is viscosity and $f(K \alpha)$ is Henry's function. Electrophoretic determinations of zeta-potential are most commonly made in aqueous media and moderate electrolyte concentration, thus, $f(K \alpha)$ in this case is $\mathbf{1 . 5}$ (referred to as the Smoluchowski approximation). The cell temperature was set to $25{ }^{\circ} \mathrm{C}$, the zetapotential measured in triplicate (based on twenty measurements per sample) for each CCNF of specific DS and the average value reported.

Small angle neutron scattering (SANS). SANS measurements were carried out on the Sans2d beamline at the ISIS Neutron and Muon Source, Science and Technology Facilities Council, Rutherford Appleton Laboratory, Didcot, UK. ${ }^{41}$ Hydrogels were placed in a $1 \mathrm{~mm}$ thick, $1 \mathrm{~cm}$ wide quartz cell with Teflon stoppers, holding $\sim 0.3 \mathrm{~mL}$. The $Q$ range measured was $0.0046-$ $1.3634 \AA$ A. To probe the structure of CCNF gels, contrast matching SANS experiments were performed using $\mathrm{CCNF} / \mathrm{H}_{2} \mathrm{O}$ and $\mathrm{CCNF} /$ $\mathrm{D}_{2} \mathrm{O}$. Scattering patterns were reduced using routines in the Mantid software package to correct for the scattering of air and the sample holder (rendering the data instrument independent) and a background of $\mathrm{H}_{2} \mathrm{O} / \mathrm{D}_{2} \mathrm{O}$ subtracted before the data was analysed using SaSview 2.1 fitting software. Various models were tested, beginning with a simple rigid cylinder, but the best fit was found to be a flexible, ellipsoidal cylinder model. The flexible, ellipsoidal cylinder model is described in full by Chen et $a .^{42}$ and Dreiss. ${ }^{43}$ In short, this model is of a number of locally stiff, ellipsoidal cross-section rods, defined by the cross-section minor radius, $r_{\mathrm{a}}$, the axis ratio, $r_{\mathrm{b}} / r_{\mathrm{a}}$, persistence length, $l_{\mathrm{p}}$, and the contour length, $L$ (Fig. 1). The Kuhn length, $b=2 \times l_{\mathrm{p}}$, is a measure of stiffness. Scattering length densities (SLDs) were fixed (cellulose $1.76 \times 10^{-6}, \mathrm{H}_{2} \mathrm{O}-5.62 \times 10^{-7}, \mathrm{D}_{2} \mathrm{O} 6.34 \times 10^{-6} \AA^{-2}$ ) and, as the aspect ratios of CNF are very large, ${ }^{44}$ fibril length does not impact scattering patterns in the $Q$ range accessed, thus $L$ was fixed at $10000 \AA$ (full fitting parameters, Table S1, ESI

\section{Results}

\section{Surface modification and characterisation}

The nucleophilic substitution of alkali activated primary alcohol groups exposed on the cellulose surface with GTMAC resulted in cationisation of the nanofibrils, leading to a positive surface charge (Scheme 1, reaction d). Increasing molar ratio of GTMAC: AGU resulted in proportionally higher DS (from $10.6 \%$ at 0.5 mole eq. GTMAC to $23.0 \%$ at 3 mole eq. GTMAC). A yield

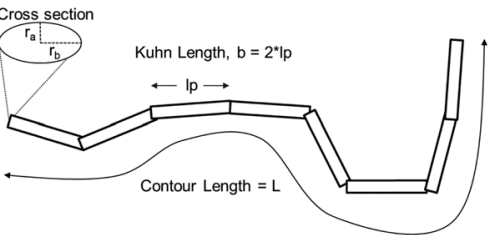

Fig. 1 Schematic of the flexible, ellipsoidal cylinder model as applied to CCNF. The Kunn length was used to describe the stiffness of the nanofibrils dispersed in the hydrogel. ${ }^{45}$

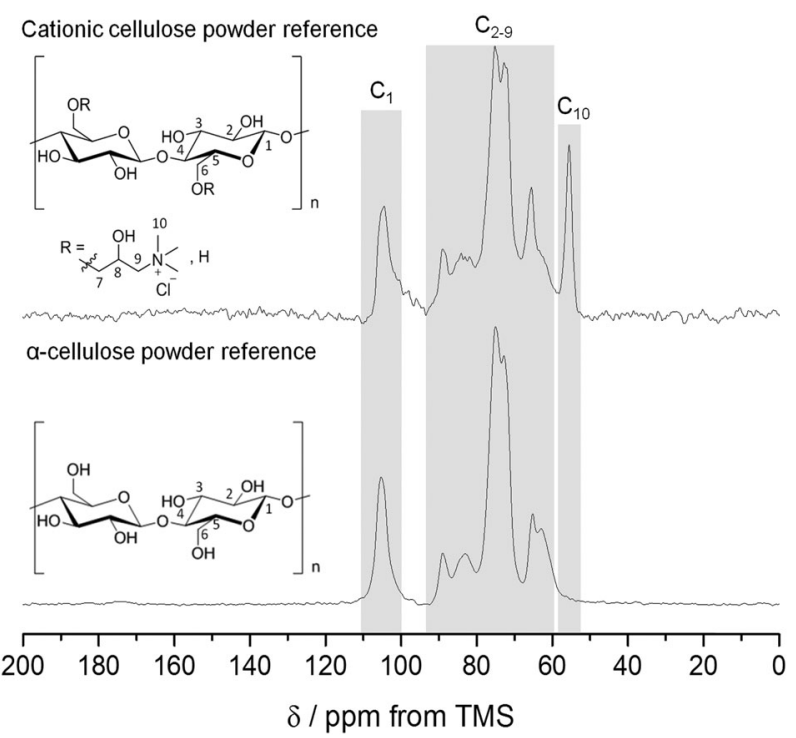

Fig. $2{ }^{1} \mathrm{H}-{ }^{13} \mathrm{C}$ CP/MAS NMR spectra for $\alpha$-cellulose and CCNF (DS 23.0\%) powders, acquired using MAS rates of $10 \mathrm{kHz}$. The peak at $55.5 \mathrm{ppm}$ is assigned to the methyl carbon resonances of the quaternary ammonium group and used to determine DS.

of $88 \%$, based on cellulose dry mass, was achieved for the cationisation process at the highest DS. The degree of surface modification was controlled by modulating the quantity of GTMAC and the DS of cellulose nanofibrils assessed using FTIR and conductometric titration (Fig. S1-S3, ESI $\$$ ). Solid-state NMR spectroscopy was used to confirm the presence of the $-\mathrm{N}^{+}\left(\mathrm{CH}_{3}\right)_{3}$ groups, introduced by reaction with GTMAC, on the surface of the nanofibrils (carbon $\mathrm{C}_{10}$ in Fig. 2). Moreover, a very good agreement was found between the DS calculated from conductometric and NMR spectroscopy experiments (Fig. 3). The CCNF zeta-potential increased upon cationisation from 38.8 to $50.3 \mathrm{mV}$ as the $-\mathrm{N}^{+}\left(\mathrm{CH}_{3}\right)_{3}$ groups graft to the surface (Fig. 3). Additionally, comparison of ${ }^{13} \mathrm{C}-\mathrm{NMR}$ signals, arising from $\mathrm{C} 4$, in crystalline and amorphous forms, revealed no discernable change in crystallinity upon cationisation ${ }^{46}$ (crystallinity index $=0.38$ ) (Fig. S4, ESI ).

\section{Characterisation of functionalised nanofibrils}

To compare the effect of DS of the CCNF on the structure of the dispersed CCNF gel, SANS with contrast matching was employed. This is a non-invasive technique used to obtain structural information from the samples in situ, based on SLD differences. For each 


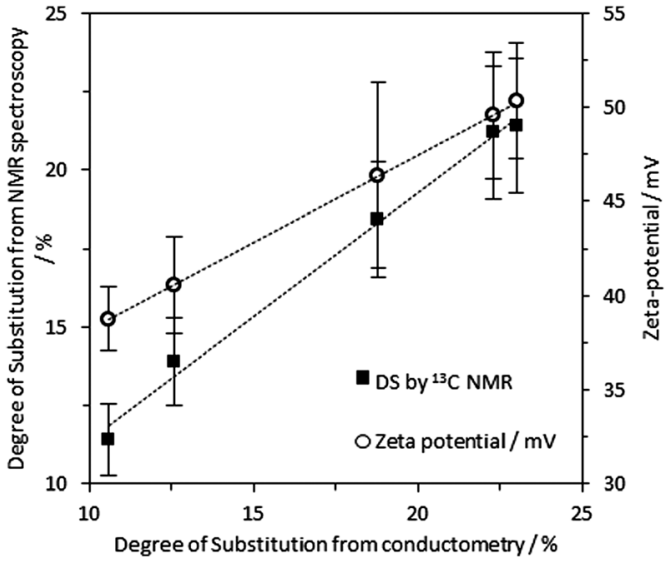

Fig. 3 Black squares: DS determined from ${ }^{13} \mathrm{C}$ solid state NMR spectra according to eqn (3) is in good agreement with the DS determined by conductometry (trend line $R^{2}=0.99$ ). White circles: zeta-potential for dispersed $\mathrm{CCNF}$ in $\mathrm{DI} \mathrm{H}_{2} \mathrm{O}$ as DS increases (trend line $R^{2}=0.99$ and gradient $=0.93$.

CCNF hydrogel system, the $\mathrm{H}_{2} \mathrm{O} / \mathrm{D}_{2} \mathrm{O}$ contrasts were plotted and the model simultaneously fitted to both sets of SANS data (Fig. 4 and Fig. S6-S9, ESI\$). The resultant ellipsoidal cross-section flexible cylinder model provided information pertaining to CCNF cross section and fibril stiffness, Table 1.

The CCNF cross-section dimensions were observed to change with increasing DS. The minor radius, $r_{\mathrm{a}}$, derived from the model changes from $1.4 \mathrm{~nm}$ to $0.6 \mathrm{~nm}$ and the axis ratio, $r_{\mathrm{b}} / r_{\mathrm{a}}$, from 2.1 to 6.6 , giving fibril cross-section dimensions of $2.8 \times 5.9 \mathrm{~nm}$ for CCNF with DS $=10.6 \%$ and $1.2 \times 8.3 \mathrm{~nm}$ for CCNF with DS $=23.0 \%$. These dimensions are in broad agreement with previous reports ${ }^{38}$ and the changes imply that the fibrils become wider and flatter as surface derivatisation increased. An increase in the Kuhn length from 18 to $30 \mathrm{~nm}$ (Table 1 and Fig. 5) was observed with increasing DS, ascribed to "stiffening" of the

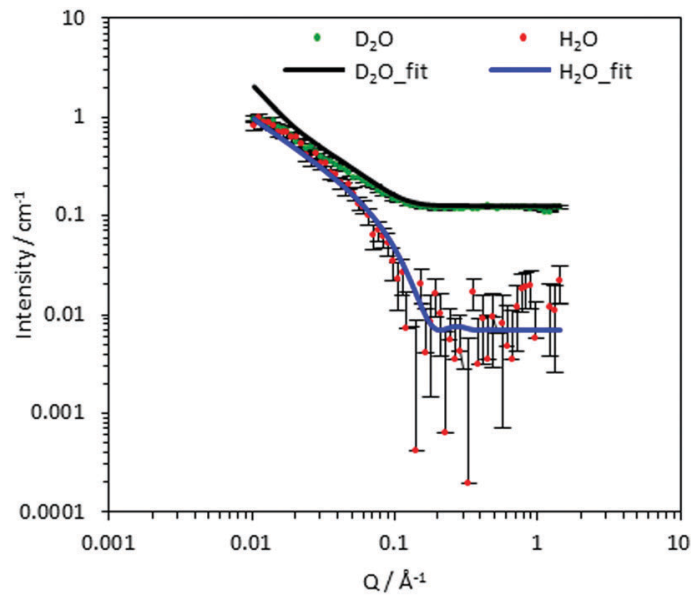

Fig. 4 Experimental SANS spectra of cationic cellulose hydrogels $(10.6 \%$ DS) fitted to a flexible ellipsoidal cylinder model (scattered points are measured data and solid lines are the simultaneously fitted curves) in $\mathrm{D}_{2} \mathrm{O}$ (green points, black line) and $\mathrm{H}_{2} \mathrm{O}$ (red points, blue line). This model describes the CCNF particles as flexible fibrils.
Table 1 Summary of fitted parameters for nanofibrils with increasing \% DS derived from simultaneously fitting $\mathrm{D}_{2} \mathrm{O}$ and $\mathrm{H}_{2} \mathrm{O}$ contrasts to a flexible ellipsoidal cylinder model ${ }^{a}$

\begin{tabular}{|c|c|c|c|}
\hline DS/\% & Radius/Å & Axis ratio & Kuhn length/Å \\
\hline $10.6(3)$ & $14(1)$ & $2.1(3)$ & 175(8) \\
\hline $12.6(1)$ & $11.6(8)$ & $3.2(6)$ & $230(14)$ \\
\hline $18.8(2)$ & $6.7(8)$ & $8.4(2)$ & $303(22)$ \\
\hline $23.0(9)$ & $6.3(8)$ & $6.6(1)$ & $296(21)$ \\
\hline
\end{tabular}

${ }^{a}$ Parameters held constant were: background, contour length (10 $000 \AA$ A), sldCyl $\left(1.75 \times 10^{-6} \AA^{-2}\right)$ and sldSolv $\left(\mathrm{D}_{2} \mathrm{O}=6.34 \times 10^{-6}\right.$ and $\mathrm{H}_{2} \mathrm{O}=$ $-5.61 \times 10^{-7} \AA^{-2}$ ).

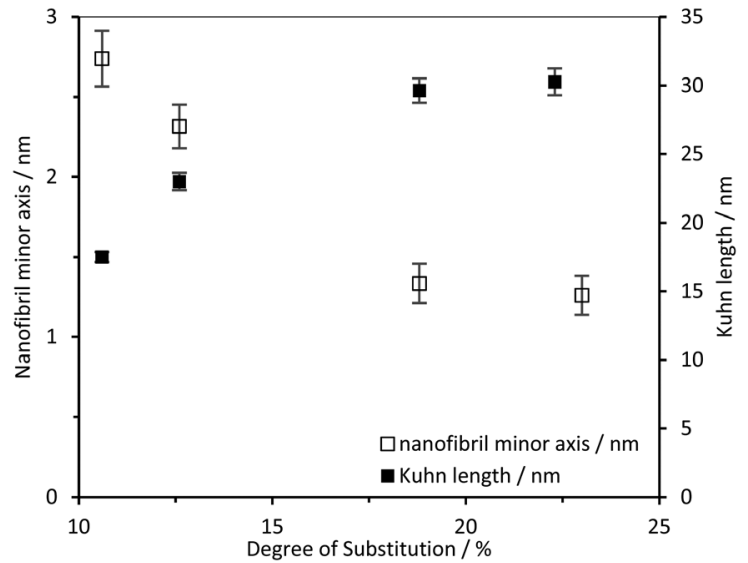

Fig. 5 CCNF dimensions determined from the simultaneously fitted $\mathrm{D}_{2} \mathrm{O} / \mathrm{H}_{2} \mathrm{O}$ data to the model nanofibril diameter and nanofibril Kuhn length.

fibrils arising from charge repulsion as the density of surface $-\mathrm{N}^{+}\left(\mathrm{CH}_{3}\right)_{3}$ groups increased. We return to discussion of the implication of the apparent flattening discerned from SANS measurements later.

\section{CCNF hydrogel rheology}

The rheological properties of the hydrogels formed by dispersion and sonication of CCNF in water were measured as a function of varying DS using both oscillatory sweep and thixotropic loop experiments. It was apparent that, as the CCNF DS increased, the hydrogels became stiffer (Fig. 6a). Furthermore, a relationship between hydrogel elastic storage modulus and Kuhn length was observed, (Fig. 6b), pointing to a change in the nature of the hydrogel associated with changes in shape and stiffness of the CCNF.

Complex modulus increased for hydrogels formed from CCNF with greater DS, while phase angle decreased (Table 2 and Fig. S10-13, ESI‡), i.e. gels became more elastic.

\section{Probing local mobility of surface functional groups using ${ }^{1} \mathrm{H}$ NMR relaxation measurements}

${ }^{1} \mathrm{H}$ longitudinal relaxation times $\left(T_{1}\right)$ of the hydrogels formed were measured as a function of varying DS, using solution-state and HR-MAS NMR experiments. Signals arising from the ${ }^{1} \mathrm{Hs}$ of the $-\mathrm{N}^{+}\left(\mathrm{CH}_{3}\right)_{3}$ moiety were used for quantification as these were distinct, while other signals were overlapped (Fig. S14-16, ESI‡). Relaxation profiles of these ${ }^{1} \mathrm{Hs}$ are affected by molecular 
a)

Table 2 Summary of hydrogel rheology for cationised cellulose. DS values; complex $\left(G^{\star}\right)$, elastic $\left(G^{\prime}\right)$ and viscous $\left(G^{\prime \prime}\right)$ moduli; phase angles $(\delta)$; viscosities $(\eta)$; and yield stresses $\left(\tau^{0}\right)$ of the aqueous gels obtained. Oscillatory rheological data were obtained for 2 wt\% hydrogels at $1.02 \mathrm{~Hz}$ and shear sweep data at shear rate $113.17 \mathrm{~s}^{-1}$

\begin{tabular}{lcrcclr}
\hline $\mathrm{DS} / \%$ & $G^{*} / \mathrm{Pa}$ & \multicolumn{1}{c}{$\delta /^{\circ}$} & $G^{\prime} / \mathrm{Pa}$ & $G^{\prime \prime} / \mathrm{Pa}$ & $\eta / \mathrm{Pas}$ & $\tau^{0} / \mathrm{Pa}$ \\
\hline 10.6 & 8.3 & 18.6 & 7.9 & 2.7 & 0.1 & 3.8 \\
12.6 & 40.8 & 10.1 & 40.4 & 7.2 & 0.3 & 38.1 \\
18.8 & 55.3 & 7.4 & 54.8 & 7.1 & 0.3 & 49.5 \\
23.0 & 63.4 & 9.5 & 62.5 & 10.5 & 0.4 & 53.3
\end{tabular}

motions and are thus a suitable probe for the effect of DS on local viscosity. To investigate the dependence of ${ }^{1} \mathrm{H}$ longitudinal relaxation times on the mobility of the cationic groups, the hydrogel with a DS of $23.0 \%$ was subjected to varying temperatures. In the temperature range employed, ${ }^{1} \mathrm{H}$ solution-state NMR $T_{1}$ times for the $-\mathrm{N}^{+}\left(\mathrm{CH}_{3}\right)_{3}$ and water protons increased with temperature (Table S2, ESI ), indicating that these hydrogels are in the "slow tumbling regime" ${ }^{47}$ The slower relaxation profiles observed at higher temperatures reflected increased motional freedom. Comparing fibrils with variable DS: longer ${ }^{1} \mathrm{H} T_{1}$ values were recorded in hydrogels with a lower DS $(10.6 \%)$ in comparison with hydrogels with higher DS (23.0\%) (Fig. 7 and 8). Therefore, it was

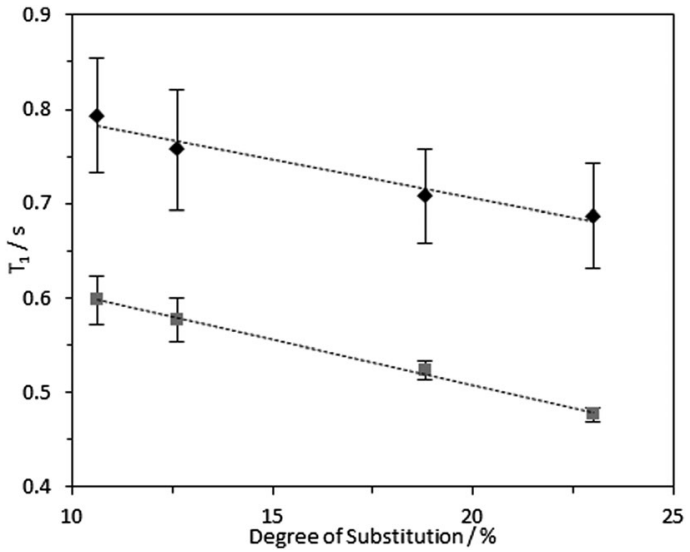

Fig. $7{ }^{1} \mathrm{H}$ solution-state (grey square data series) and HR-MAS NMR (black diamond data series) $T_{1}$ times for $-\mathrm{N}^{+}\left(\mathrm{CH}_{3}\right)_{3}$ group ${ }^{1} \mathrm{Hs}$ in $4 \mathrm{wt} \% \mathrm{CCNF}$ hydrogels with DS $10.6-23.0 \%$, acquired at $25^{\circ} \mathrm{C}$. HR-MAS experiments were conducted with an MAS rate of $2 \mathrm{kHz}$.

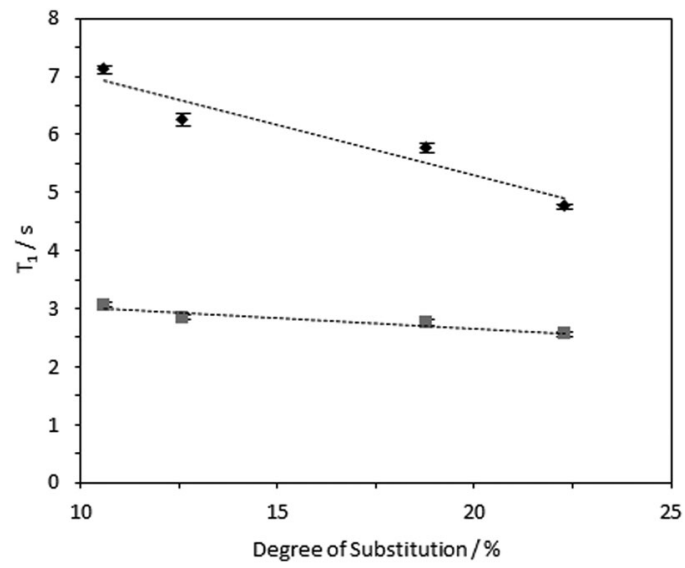

Fig. $8{ }^{1} \mathrm{H}$ solution-state (grey square data series) and HR-MAS NMR (black diamond data series) $T_{1}$ times for water ${ }^{1} \mathrm{Hs}$ in $4 \mathrm{wt} \%$ CCNF hydrogels with DS $10.6-23.0 \%$, acquired at $25{ }^{\circ} \mathrm{C}$. HR-MAS experiments were conducted with an MAS rate of $2 \mathrm{kHz}$.

possible to conclude that the quaternary ammonium groups and water molecules were more mobile in hydrogels with lower DS, showing that the local mobility of the grafted quaternary ammonium group depends on the DS.

Interestingly, an inverse relationship was found between DS and ${ }^{1} \mathrm{H}$ HR-MAS $T_{1}$ values of $-\mathrm{N}^{+}\left(\mathrm{CH}_{3}\right)_{3}$ and water acquired with different MAS rates (Fig. 9 and Fig. S17, ESI‡). While the spectra did not change dramatically (Fig. S17, ESI $\$$ ), the ${ }^{1} \mathrm{H} T_{1}$ times increased when all the samples were subjected to higher spinning speeds, possibly reflecting shear thinning behaviour in these CCNF hydrogels. This effect was more evident for hydrogels with lower DS (10.6\%). The shear force produced by the spinning rates used was significantly greater than that required to "break" the gels (as evidenced by the rheology data presented in Table 2 and Fig. S9-S12, ESI ), so it is hypothesised that there are multiple levels of CCNF interaction. When monitoring ${ }^{1} \mathrm{H} T_{1}$ times of the $-\mathrm{N}^{+}\left(\mathrm{CH}_{3}\right)_{3}$ group we are only assessing the local mobility of the groups grafted onto the surface of the fibres. 


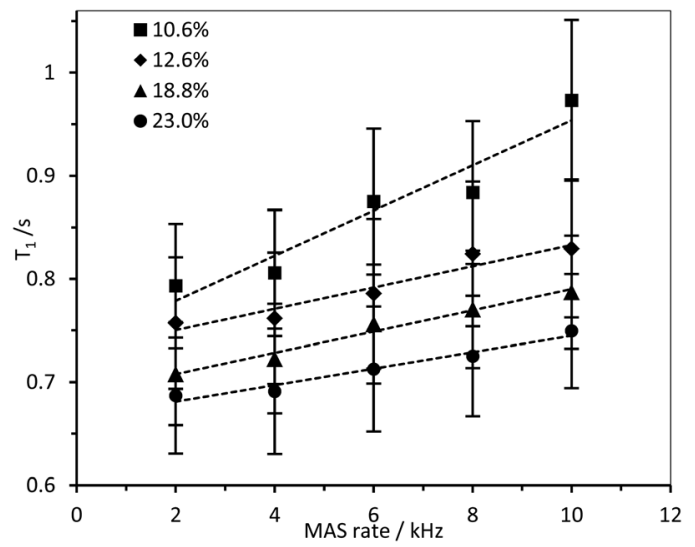

Fig. $9{ }^{1} \mathrm{H}$ HR-MAS $T_{1}$ times for $-\mathrm{N}^{+}\left(\mathrm{CH}_{3}\right)_{3}$ group in $4 \mathrm{wt} \% \mathrm{CCNF}$ hydrogels with increasing DS, acquired with MAS rates between 2 and $10 \mathrm{kHz}$.

During rheology studies these local environments do not change significantly, as it is the macrostructure and mesostructure of the gel that are most affected by the external stress. The ${ }^{1} \mathrm{H}$ relaxation time measurements suggest that, along with the changes at mesoscale reflected by the rheological properties, local mobilities of both water and grafted $-\mathrm{N}^{+}\left(\mathrm{CH}_{3}\right)_{3}$ groups on the CCNF are reduced with increased strength of the hydrogels.

\section{Discussion}

Stable hydrogels were formed upon the dispersion of cationically surface modified cellulose nanofibrils in water. The physical interactions of the surfaces of CCNF were investigated using NMR, SANS and rheology to reveal the fundamental form of the particles and structuring in the hydrogels.

The presence of tetra-alkylammonium moieties arising from grafting with GTMAC was confirmed by ${ }^{1} \mathrm{H}^{-13} \mathrm{C}$ CP/MAS solid-state NMR (Fig. 2), which revealed a peak at $55.5 \mathrm{ppm}$, corresponding to the three methyl group carbon atoms of the quaternary ammonium moiety. Very good agreement was obtained for the values of DS obtained from conductometric titration measurements (versus $\mathrm{AgNO}_{3}$, or sodium polyethylene sulfonate solutions) and quantitative NMR analysis (Fig. 3).

As previously reported ${ }^{38}$ the modified nanofibrils were much more dispersible in DI water than the $\alpha$-cellulose precursor, but remained largely water insoluble (DS was limited to $<25 \%$ to minimise formation of soluble $\mathrm{CCNF}^{48}$ ). Moreover, while all CCNF prepared formed stable hydrogels in water following dispersion by sonication, nanofibrils with higher DS were more readily dispersed and formed stiffer and more elastic gels. The linear relationship, with gradient close to unity, between DS and zeta-potential (Fig. 3) suggested that the etherification to install cationic groups was largely limited to the nanofibril surfaces. The maximum theoretical DS achievable for surface functionalisation, calculated following Habibi et al. ${ }^{16,36}$ was $c a .32 \%$, thus the CCNF produced here may not reflect full nanofibrillar surface saturation. Further, the unchanging crystallinity index suggested that the bulk structure of the elemental nanofibril remained untouched by the modification (Fig. S4, ESI ).
SANS was used to probe the CCNF cross-section and to elucidate the gel structure and nanofibril interactions. The reduced scattering data was fitted to a flexible ellipsoidal cylinder model, which describes the CCNF as a fibril with somewhat flattened crosssection and varying degrees of flexibility. Further, results suggest that charge repulsion between the positively charged nanofibril surfaces serve to facilitate defibrillation, producing individualised fibrils, which become stiffer with increasing DS, as evidenced by an increase in Kuhn length (Fig. 5) and supported by TEM images (Fig. 10), showing flexed fibrils in samples with low DS and distinctly straighter fibrils in high DS materials. As with oxidised cellulose nanofibrils, the very high fibrillar aspect ratio ${ }^{44}$ provides a particulate network, forming hydrogels. The nanofibrillar cross-section dimensions measured are consistent with values reported for cationic cellulose nanofibrils, determined by AFM and Cryo-TEM ${ }^{38}$ and the theoretical range of unmodified cellulose crystallites of $1.5-2 \mathrm{~nm}$ by 3-5 $\mathrm{nm}$, based on the 36 -glucan-chain elementary model of the cellulose $\mathrm{I} \beta$ structure, ${ }^{49}$ although the 24-glucan chain elementary fibril postulated by Fernandes et $a .^{50}$ could lead to thinner forms.

The level of surface modification present on the dispersed nanofibrils influenced the rheology of the resultant hydrogels. CCNF with higher DS formed stiffer hydrogels reflected in higher elastic storage moduli (Fig. 6a and Table 2). As this change in rheological behaviour was also mirrored in the increased stiffness of the dispersed nanofibrils (Kuhn length) and a change in apparent cross-section, it is postulated that this change in hydrogel structure arises from:

1. Increased charge repulsion with increased DS leading to loss of flexibility and straightening and stiffening of fibrils along their length - reflected in longer Kuhn lengths and decreased mobility of surface $-\mathrm{N}^{+}\left(\mathrm{CH}_{3}\right)_{3}$ groups, and

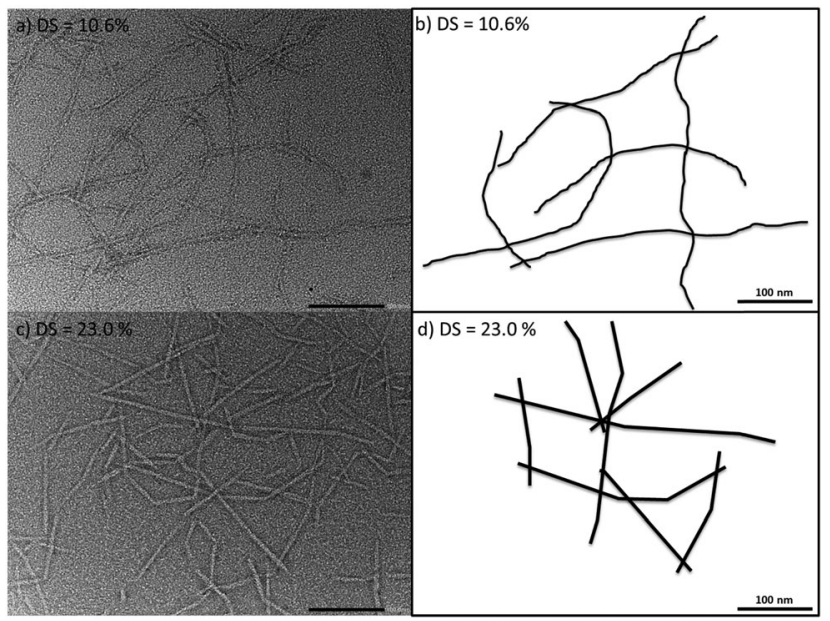

Fig. 10 TEM images of CCNF after dispersion in water ( $a$ and $c$ ) and traces of selected fibrils ( $b$ and $d$ ). Fibril cross-section diameters are similar to those derived from SANS data fitting. (a) Low DS $=10.6 \%$, scale bar $=$ $100 \mathrm{~nm}$, (b) trace of low DS CCNF, (c) high DS $=23.0 \%$, scale bar $=100 \mathrm{~nm}$ and (d) trace of high DS CCNF. As DS increases, a change in the morphology of the CCNF is observed with low DS fibrils appearing more flexed and higher DS fibrils straighter. The traces are provided to highlight the change in morphology between the two CCNF. 
2. Untwisting, or unravelling, of CCNF to form flatter, more ribbon-like structures at higher DS, reflected in changes in cross-section shape and diameter - surface charge repulsion caused CCNF to untwist into flattened ribbon-like fibrils as DS increased.

The changes in rheological behaviour of CCNF hydrogels with different DS can be correlated with the changes in local mobility of grafted quaternary ammonium groups and water molecules monitored by ${ }^{1} \mathrm{H} T_{1}$ relaxation times. Thus, low DS CCNF gels result from entanglement of long twisted flexible fibrils, which become less twisted and more rigid as DS increases (Fig. 10). Entanglement of these stiffer units leads to stiffer, more elastic hydrogels.

It is well documented that cellulose nanofibrils possess a twist along the AGU chain axis: computational modelling (molecular dynamics) has been used to demonstrate that cellulose nanofibrils adopt a half twist every 3-4 $\mathrm{nm}$ along the chain axis; ${ }^{51-53}$ this twisting has also been observed by AFM and TEM analyses and is especially apparent in well-dispersed ribbon-shaped bacterial cellulose. ${ }^{54,55}$ We suggest that the introduction of a positive surface onto the cellulose causes nanofibrils to untwist due to charge repulsion along the surface (Fig. 11). Furthermore the values for Kuhn length $(18-30 \mathrm{~nm})$ are similar to those previously reported for cellulose $\sim 10-20 \mathrm{~nm}$ (using a similar model) ${ }^{56}$ and $\sim 50-60 \mathrm{~nm}$ for oxidised cellulose nanofibrils (determined from peak force quantitative nanomechanical mapping). ${ }^{57}$

This hypothesis for the apparent change in CCNF structure is supported by the rise in hydrogel elastic moduli of 7.9-62.5 Pa. These hydrogels are highly thixotropic as the viscosity decreases rapidly as the shear rate increases. Increasing the oscillation frequency gave increased complex modulus and decreased phase angle values, associated with increasing elastic behaviour with decreasing perturbation time. As the DS increased, both the

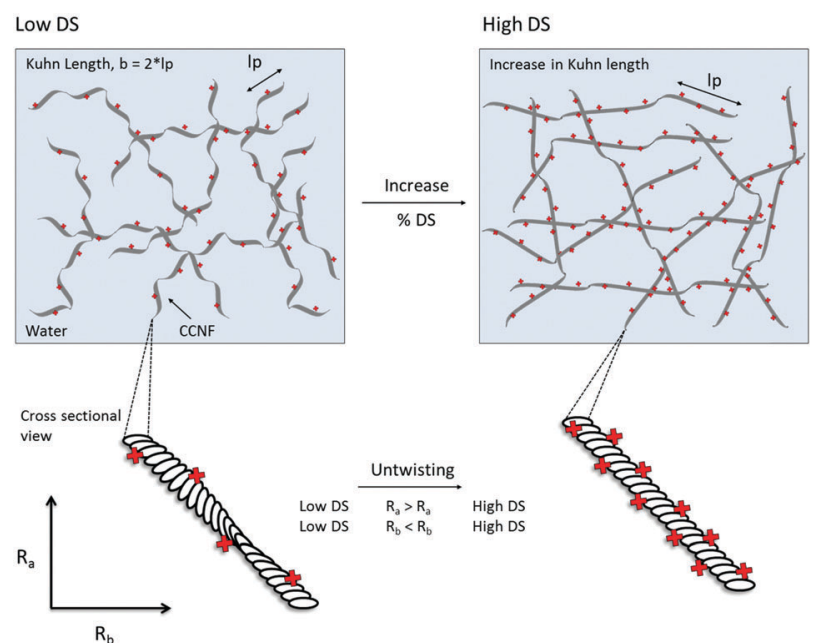

Fig. 11 A schematic illustrating the postulated change in CCNF morphology as DS increases, reflecting SANS data fitting and TEM images. As the DS on the surface of the nanofibrils increases, so too does the Kuhn length, resulting in more rigid nanofibrils. The CCNF also untwist to form more flattened ribbon-like nanofibrils - stiffer, more elastic hydrogels result $\left(l_{p}=\right.$ persistence length and $2 \times l_{p}=$ Kuhn length). elastic and viscous moduli values increased, but the elastic modulus, already much higher, increased to a greater extent, which is a good indication of structuring within the hydrogel.

\section{Conclusions}

Combining results from neutron scattering experiments, rheological measurements and analysis of ${ }^{1} \mathrm{H}$ NMR $T_{1}$ relaxation times of the water and grafted $-\mathrm{N}^{+}\left(\mathrm{CH}_{3}\right)_{3}$ groups, changes in the form of cationised cellulose nanofibrils and structuring in hydrogels formed therefrom is revealed.

With increased degree of reaction with GTMAC, the density of positively charged moieties on the surface of the CCNF increases, leading to charge repulsion and stiffening of the fibrils. Physical entanglement of these stiffer CCNF results in stiffer, but more fragile, gels. Changes in the apparent crosssection dimensions of the individualised fibrils, derived from fitting of SANS data, are postulated to reflect untwisting of the fibrils. Thus, low DS CCNF are characterised by fibrils that are twisted and flexed, and which entangle to form weak hydrogels, while higher DS CCNF fibrils are more rigid, flatter, ribbon-like structures that result in stiff, elastic viscous shear thinning hydrogels.

\section{Conflicts of interest}

The authors declare no conflicts of interest.

\section{Acknowledgements}

We acknowledge ISIS for the provision of beamtime for the SANS experiments (experiment number RB1520375) and Dr Richard K. Heenan for their assistance in setting up the SANS2D instrument during the experiment. S. M. R. thanks the University of East Anglia for her postgraduate studentship. Y. Z. K., K. J. E. and J. L. S. acknowledge financial support from the EPRSC and Innovate UK - from the grants EP/N033337/1 and EP/N033310/1. TEM images were obtained at the Microscopy and Analysis Suite in Bath University with the assistance of Ursula Potter. J. C. C. thanks Vincenzo Calabrese for his assistance with rheology analysis. This research was partly supported by funding from the EPSRC Centre for Doctoral Training in Sustainable Chemical Technology (EP/L016354/1), in the form of a PhD studentship for J. C. C. Additional research data supporting this publication are available as electronic supplementary files at the DOI: $10.1039 / \mathrm{c} 7 \mathrm{sm02113e.}$

\section{References}

1 D. Klemm, F. Kramer, S. Moritz, T. Lindström, M. Ankerfors, D. Gray and A. Dorris, Angew. Chem., Int. Ed., 2011, 50, 5438-5466.

2 S. Kalia, S. Boufi, A. Celli and S. Kango, Colloid Polym. Sci., 2014, 292, 5-31.

3 B. G. Rånby, Discuss. Faraday Soc., 1951, 11, 158-164. 
4 F. Alloin, A. Dufresne and M. A. S. A. Samir, Biomacromolecules, 2005, 6, 612-626.

5 Y. Habibi, L. A. Lucia and O. J. Rojas, Chem. Rev., 2010, 110, 3479-3500.

6 G. Siqueira, J. Bras and A. Dufresne, Polymers, 2010, 2, 728-765.

7 R. J. Moon, A. Martini, J. Nairn, J. Simonsen and J. Youngblood, Chem. Soc. Rev., 2011, 40, 3941-3994.

8 T. Saito, S. Kimura, Y. Nishiyama and A. Isogai, Biomacromolecules, 2007, 8, 2485-2491.

9 T. Saito, Y. Nishiyama, J. L. Putaux, M. Vignon and A. Isogai, Biomacromolecules, 2006, 7, 1687-1691.

10 T. Saito, T. Uematsu, S. Kimura, T. Enomae and A. Isogai, Soft Matter, 2011, 7, 8804.

11 X. Shen, J. L. Shamshina, P. Berton, G. Gurau and R. D. Rogers, Green Chem., 2015, 18, 53-75.

12 R. J. Crawford, K. J. Edler, S. Lindhoud, J. L. Scott and G. Unali, Green Chem., 2012, 14, 300-303.

13 J. C. Courtenay, M. A. Johns, F. Galembeck, C. Deneke, E. M. Lanzoni, C. A. Costa, J. L. Scott and R. I. Sharma, Cellulose, 2017, 24, 253-267.

14 J. You, J. Cao, Y. Zhao, L. Zhang, J. Zhou and Y. Chen, Biomacromolecules, 2016, 17, 2839-2848.

15 A. Pei, N. Butchosa, L. A. Berglund and Q. Zhou, Soft Matter, 2013, 9, 2047.

16 T. Saito and A. Isogai, Biomacromolecules, 2004, 5, 1983-1989.

17 M. Zaman, H. Xiao, F. Chibante and Y. Ni, Carbohydr. Polym., 2012, 89, 163-170.

18 Y. Habibi, Chem. Soc. Rev., 2014, 43, 1519-1542.

19 C. Goussé, H. Chanzy, G. Excoffier, L. Soubeyrand and E. Fleury, J. Polym., 2002, 43, 2645-2651.

20 J. F. Sassi and H. Chanzy, Cellulose, 1995, 2, 111-127.

21 Y. Habibi, A.-L. Goffin, N. Schiltz, E. Duquesne, P. Dubois and A. Dufresne, J. Mater. Chem., 2008, 18, 5002-5010.

22 J. K. Pandey, W. S. Chu, C. S. Kim, C. S. Lee and S. H. Ahn, Composites, Part B, 2009, 40, 676-680.

23 A. Junior de Menezes, G. Siqueira, A. A. S. Curvelo and A. Dufresne, J. Polym., 2009, 50, 4552-4563.

24 G. Siaueira, J. Bras and A. Dufresne, Biomacromolecules, 2009, 10, 425-432.

25 S. P. Akhlaghi, R. C. Berry and K. C. Tam, Cellulose, 2013, 20, 1747-1764.

26 C. Chang and L. Zhang, Carbohydr. Polym., 2011, 84, 40-53.

27 M. Hasani, E. D. Cranston, G. Westman and D. G. Gray, Soft Matter, 2008, 4, 2238-2244.

28 A. Isogai, T. Saito and H. Fukuzumi, Nanoscale, 2011, 3, 71-85.

29 M. Hasani, E. D. Cranston, G. Westman and D. G. Gray, Soft Matter, 2008, 4, 2238-2244.

30 J. L. Ren, R. C. Sun, C. F. Liu, Z. Y. Chao and W. Luo, Polym. Degrad. Stab., 2006, 91, 2579-2587.

31 A. Isogai, T. Saito and H. Fukuzumi, Nanoscale, 2011, 3, 71-85.

32 D. da Silva Perez, S. Montanari and M. R. Vignon, Biomacromolecules, 2003, 4, 1417-1425.
33 H. Kono, K. Ogasawara, R. Kusumoto, K. Oshima, H. Hashimoto and Y. Shimizu, Carbohydr. Polym., 2016, 152, 170-180.

34 R. J. Crawford, K. J. Edler, S. Lindhoud, J. L. Scott and G. Unali, Green Chem., 2012, 14, 300-303.

35 K. Edler, D. Celebi, Y. Jin and J. Scott, Acta Crystallogr., Sect. A: Found. Adv., 2014, 70, C1320.

36 Y. Habibi, H. Chanzy and M. R. Vignon, Cellulose, 2006, 13, 679-687.

37 N. Masruchin, B.-D. Park, V. Causin and I. C. Um, Cellulose, 2015, 22, 1993-2010.

38 A. Olszewska, P. Eronen, L.-S. Johansson, J.-M. Malho, M. Ankerfors, T. Lindström, J. Ruokolainen, J. Laine and M. Österberg, Cellulose, 2011, 18, 1213-1226.

39 R. Pelton, B. Cabane, Y. Cui and H. Ketelson, Anal. Chem., 2007, 79, 8114-8117.

40 W. Kolodziejski and J. Klinowski, Chem. Rev., 2002, 102, 613-628.

41 R. K. Heenan, S. M. King, D. S. Turner and J. R. Treadgold, 17th Meeting of the International Collaboration on Advanced Neutron Sources, 2005, pp. 1-6.

42 W. Chen, P. D. Butler and L. J. Magid, Langmuir, 2006, 22, 6539-6548.

43 C. A. Dreiss, Soft Matter, 2007, 3, 956.

44 Q. Li and S. Renneckar, Cellulose, 2009, 16, 1025-1032.

45 T. Strey, Description of the Model Functions, http://danse. chem.utk.edu/downloads/ModelfuncDocs.pdf, accessed 25 August 2015.

46 R. H. Newman, Holzforschung, 2004, 58, 91.

47 C. L. Cooper, T. Cosgrove, J. S. van Duijneveldt, M. Murray and S. W. Prescott, Soft Matter, 2013, 9, 7211-7228.

48 L. Yan, H. Tao and P. R. Bangal, Clean: Soil, Air, Water, 2009, 37, 39-44.

49 S.-Y. Ding and M. E. Himmel, J. Agric. Food Chem., 2006, 54, 597-606.

50 A. N. Fernandes, L. H. Thomas, C. M. Altaner, P. Callow, V. T. Forsyth, D. C. Apperley, C. J. Kennedy and M. C. Jarvis, Proc. Natl. Acad. Sci. U. S. A., 2011, 108, 1195-1203.

51 K. Conley, L. Godbout, M. A. Whitehead and T. G. M. Van De Ven, Carbohydr. Polym., 2016, 135, 285-299.

52 S. Paavilainen, T. Róg and I. Vattulainen, J. Phys. Chem. B, 2011, 115, 3747-3755.

53 Z. Zhao, O. E. Shklyaev, A. Nili, M. Naseer, A. Mohamed, J. D. Kubicki, V. H. Crespi and L. Zhong, J. Phys. Chem. A, 2013, 117, 2580-2589.

54 S. Elazzouzi-hafraoui, Y. Nishiyama, L. Heux, F. Dubreuil, C. Rochas and J. Putaux, Biomacromolecules, 2008, 9, 57-65.

55 S. Hanley, J.-F. Revol, L. Godbout and D. Gray, Cellulose, 1997, 4, 209-220.

56 C. G. Lopez, S. E. Rogers, R. H. Colby, P. Graham and J. T. Cabral, J. Polym. Sci., Part B: Polym. Phys., 2015, 53, 492-501.

57 I. Usov, G. Nyström, J. Adamcik, S. Handschin, C. Schütz, A. Fall, L. Bergström and R. Mezzenga, Nat. Commun., 2015, 6, 7564 . 